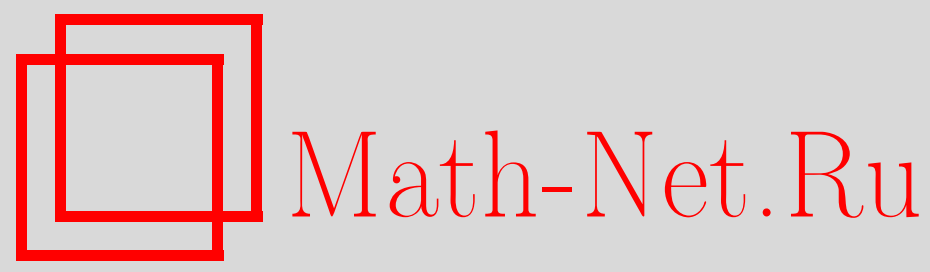

М. К. Кравцов, О. А. Янушкевич, О разрешимости векторной задачи с помощью алгоритма линейной свертки критериев, Матем. заметки, 1997, том 62, выпуск 4, 502-509

DOI: https://doi.org/10.4213/mzm1633

Использование Общероссийского математического портала Math-Net.Ru подразумевает, что вы прочитали и согласны с пользовательским соглашением http://www . mathnet.ru/rus/agreement

Параметры загрузки:

IP : 3.80 .253 .173

26 апреля 2023 г., 14:08:43

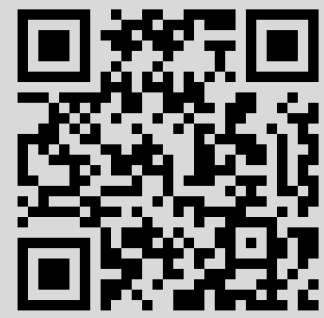




\title{
О РАЗРЕШИМОСТИ ВЕКТОРНОЙ ЗАДАЧИ С ПОМОЩЬЮ АЛГОРИТМА ЛИНЕЙНОЙ СВЕРТКИ КРИТЕРИЕВ
}

\author{
М.К. Кравцов, О.А. Янушкевич
}

\begin{abstract}
Найдены условия, при выполнении которых многокритериалная задача с конечньм множеством векторных оценок разрешима с помошью алгоритма линейной свертки критериев (ЛСК), т.е. любой паретовский оптимум задачи может быть получен как оптимальное решение однокритериалной задачи с агрегированным критерием, представляющим собой ЛСК. Предложен также полиномиальньй относительно размерности задачи алгоритм, позволяющий сводить любую задачу с минимаксными и миниминньми критериями к эквивалентной векторной задаче с тем же паретовским множеством, разрешимой с помощью алгоритма ЛСК.
\end{abstract}

Библиограффия: 15 названий.

В [1] доказано, что всякий лексикографический оптимум в задаче векторной оптимизации на конечном множестве допустимых решений может быть получен с помощью алгоритма линейной свертки критериев (ЛСК).

В настоящей работе исследуется возможность нахождения всех элементов паретовского множества в многокритериальной (векторной) задаче с конечным множеством векторных оценок. Найдены условия, при вьполнении которых такая задача разрешима с помощью алгоритма ЛСК, т.е. любой паретовский оптимум задачи может быть получен как оптимальное решение задачи с одним агрегированньм критерием, представляющим собой ЛСК. Предложен также полиномиальньй относительно размерности задачи алгоритм, позволяющий сводить любую задачу с критериями вида MIN MAX (критерий “узкого места") и MIN MIN (миниминньй критерий) к векторной задаче с тем же паретовским множеством, но разрешимой с помощью алгоритма ЛСК.

1. Признак разрешимости. Пусть на множестве допустимых решений $X=\{x\}$ задана векторная целевая функция (векторньй критерий)

$$
F=\left(f_{1}, f_{2}, \ldots, f_{r}\right): X \rightarrow \mathbb{R}^{r}, \quad r \geqslant 2
$$

с частньпи критериями

$$
f_{i}(x) \rightarrow \min _{X} \quad \forall i \in N_{r}=\{1,2, \ldots, r\}
$$

Работавыполнена при поддержке фонда фундаментальных исследований Республики Беларусь, гранты $995-70$ и МП96-35. 
Отметим, что под множеством допустимых решений $X$ будем понимать множество произвольной природы, состоящее, например, из траекторий, векторов, матриц и т.д.; $r$-критериальную задачу нахождения паретовского множества

$$
\widetilde{X}=\left\{x \in X: \bar{\exists} x^{\prime} \in X \quad F(x) \geqslant F\left(x^{\prime}\right), F(x) \neq F\left(x^{\prime}\right)\right\}
$$

будем обозначать через $(X, F)$.

Известно [2]-[6], что для любой $r$-критериальной задачи $(X, F)$ справедливо включение

$$
X^{\circ} \subseteq \tilde{X}
$$

где $X^{\circ}=\left\{X(\lambda): \lambda \in \Lambda_{r}\right\}$

$$
\begin{aligned}
& \Lambda_{r}=\left\{\lambda=\left(\lambda_{1}, \lambda_{2}, \ldots, \lambda_{r}\right): \sum_{i=1}^{r} \lambda_{i}=1, \lambda_{i}>0 \forall i \in N_{r}\right\}, \\
& X(\lambda)=\arg \min \{\Phi(x, \lambda): x \in X\}, \quad \Phi(x, \lambda)=\sum_{i=1}^{r} \lambda_{i} f_{i}(x) .
\end{aligned}
$$

Установлено существование таких многокритериальных задач, для которых включение (1) является строгим (см., например, [7]-[9]).

Будем говорить, что $r$-критериальная задача $(X, F)$ разрешима с помощью алгоритма ЛСК, если выполняется равенство $X^{\circ}=\widetilde{X}$.

В дальнейшем, говоря об $r$-критериальной задаче $(X, F)$, будем предполагать, что множество векторных оценок $Y=F(X)$ является конечным.

Для доказательства теоремы о разрешимости нам понадобится следующая лемма.

ЛЕмма. Если множсество векторных оценок $Y$-критериальной задачи $(X, F)$ конечно, то справедливо включение $X^{*} \subseteq X^{\circ}$, где

$$
X^{*}=\{x \in X: \bar{\exists} y \in \operatorname{conv} Y F(x) \geqslant y, F(x) \neq y\}
$$

ДоказАТЕЛЬСтво. Так как множество векторных оценок $Y$ конечно, то выпуклая оболочка множества $Y$, являясь вьпуклым многогранником в $\mathbb{R}^{r}$, представляет собой в силу теоремы Вейля-Минковского (см., например, [10, гл. 1, теорема 2.5]) ограниченный полиэдр

$$
\operatorname{conv} Y=\left\{y \in \mathbb{R}^{r}: A y \geqslant b\right\},
$$

где $A \in \mathbb{R}^{m \times r}, b \in \mathbb{R}^{m}, m \in \mathbb{N}$.

Для произвольного элемента $x^{*} \in X^{*}$ определим вектор

$$
\lambda\left(x^{*}\right)=\frac{A^{T} \widehat{v}}{\left\langle A^{T} \widehat{v}, e\right\rangle},
$$

где $\widehat{v}$ - оптимальное решение задачи линейного программирования

$$
\left\langle A F\left(x^{*}\right)-b, v\right\rangle \rightarrow \min , \quad A^{T} v \geqslant e, \quad v \geqslant 0
$$


$\langle\cdot, \cdot\rangle$ - скалярное произведение векторов, $e=(1,1, \ldots, 1) \in \mathbb{R}^{r}$. Очевидно, что вектор $\lambda\left(x^{*}\right) \in \Lambda_{r}$.

Чтобы доказать, что $x^{*} \in X^{\circ}$, достаточно обосновать неравенство

$$
\Phi\left(x^{*}, \lambda\left(x^{*}\right)\right) \leqslant \Phi\left(x, \lambda\left(x^{*}\right)\right) \quad \forall x \in X
$$

Рассмотрим задачу, двойственную к задаче (2),

$$
\langle e, u\rangle \rightarrow \max , \quad A u \leqslant A F\left(x^{*}\right)-b, \quad u \geqslant 0 .
$$

Осуществив замену $u=F\left(x^{*}\right)-y$, приходим к задаче

$$
\left\langle e, F\left(x^{*}\right)-y\right\rangle \rightarrow \max , \quad A y \geqslant b, \quad y \leqslant F\left(x^{*}\right) .
$$

Поскольку всякое допустимое решение $y$ задачи (5) содержится в conv $Y$, то заключаем (ввиду $x^{*} \in X^{*}$ ), что для ее оптимального решения $\widehat{y}$ справедливо равенство $\widehat{y}=F\left(x^{*}\right)$. Поэтому оптимальным решением задачи (4) является вектор $\widehat{u}=0$. Отсюда в силу двойственности задач $(2)$ и (4) имеем $\left\langle A F\left(x^{*}\right)-b, \widehat{v}\right\rangle=\langle e, \widehat{u}\rangle=0$. Поэтому из неравенства $A y \geqslant b$ находим

$$
\begin{gathered}
\left\langle A F\left(x^{*}\right), \widehat{v}\right\rangle=\langle b, \widehat{v}\rangle \leqslant\langle A y, \widehat{v}\rangle \quad \forall y \in \operatorname{conv} Y, \\
\left\langle A^{T} \widehat{v}, F\left(x^{*}\right)\right\rangle \leqslant\left\langle A^{T} \widehat{v}, F(x)\right\rangle \quad \forall x \in X .
\end{gathered}
$$

Поэтому с учетом выбора вектора $\lambda\left(x^{*}\right)$ имеют место неравенства $(3)$, т.е. $x^{*} \in X^{\circ}$. Лемма доказана.

Ранее лемма в несколько иной форме была сформулирована в [11].

Для всякого $i \in N_{r}$ упорядочим по возрастанию все различные значения целевой функции $f_{i}(x)$ на множестве $X$ :

$$
f_{i}\left(x_{1}^{i}\right)<f_{i}\left(x_{2}^{i}\right)<\cdots<f_{i}\left(x_{m_{i}}^{i}\right), \quad m_{i} \leqslant|Y|
$$

Не нарушая общности, можно считать, что

$$
m_{i}>1 \quad \forall i \in N_{r}
$$

Далее для всякого $i \in N_{r}$ положим $\Delta_{i}(k, l)=f_{i}\left(x_{k}^{i}\right)-f_{i}\left(x_{l}^{i}\right)$.

Теорема 1. Для того чтобы $r$-критериальная задача $(X, F)$ с конечным множеством векторных оценок $Y$ была разрешима с помощью алгоритма ЛСК достаточно, чтобы для любого $i \in N_{r}$ выполнялись неравенства

$$
r \Delta_{i}(p+1,1) \leqslant \Delta_{i}(p+2,1) \quad \forall p \in N_{m_{i}-2} .
$$


ДокАЗАТЕЛЬСтво. Согласно (1) для доказательства теоремы достаточно показать включение $\widetilde{X} \subseteq X^{\circ}$. Доказательство проведем от противного, т.е. пусть существует элемент

$$
\widetilde{x} \in \widetilde{X} \backslash X^{\circ}
$$

Тогда в силу леммы существует вектор

$$
y^{\circ} \in\{y \in \operatorname{conv} Y: F(\widetilde{x}) \geqslant y, F(\widetilde{x}) \neq y\},
$$

удовлетворяющий условию

$$
\bar{\exists} y \in \operatorname{conv} Y \quad y^{\circ} \geqslant y, \quad y^{\circ} \neq y .
$$

Отсюда, очевидно, что вектор $y^{\circ}$ принадлежит некоторой грани $G, \operatorname{dim} G \leqslant r-1$, многогранника conv $Y$. Поэтому на основании соотношений $G=\operatorname{conv} \operatorname{vert} G, \operatorname{vert} G \subseteq Y$ (см. [10, гл. 1, теорема 2.2 и следствие 2.4]) и теоремы Каратеодори (см. [10, гл. 1, теорема 1.8]) вектор $y^{\circ}$ представим в виде

$$
y^{\circ}=\sum_{j=1}^{k} \alpha_{j} F\left(x_{j}\right), \quad k \leqslant r,
$$

где $x_{j} \in X,\left(\alpha_{1}, \alpha_{2}, \ldots, \alpha_{k}\right) \in \Lambda_{k}$.

Далее, с учетом (8) для любого числа $j \in N_{k}$ существуют индекс $i(j) \in N_{r}$ и числа $p, g \in N_{m_{i(j)}}, p<g$, удовлетворяющие соотношениям

$$
f_{i(j)}\left(x_{p}^{i(j)}\right)=f_{i(j)}(\widetilde{x})<f_{i(j)}\left(x_{j}\right)=f_{i(j)}\left(x_{g}^{i(j)}\right) .
$$

Отсюда, принимая во внимание соотношения (9) и (10), выводим

$$
\begin{aligned}
f_{i(j)}\left(x_{p}^{i(j)}\right) & \geqslant \sum_{s=1}^{k} \alpha_{s} f_{i(j)}\left(x_{s}\right) \geqslant \alpha_{j} f_{i(j)}\left(x_{p+1}^{i(j)}\right)+\left(1-\alpha_{j}\right) f_{i(j)}\left(x_{1}^{i(j)}\right) \\
& =\alpha_{j} \Delta_{i(j)}(p+1,1)+f_{i(j)}\left(x_{1}^{i(j)}\right) \quad \forall j \in N_{k}
\end{aligned}
$$

причем существует такой индекс $j^{*} \in N_{k}$, для которого справедливо неравенство

$$
f_{i\left(j^{*}\right)}\left(x_{p}^{i\left(j^{*}\right)}\right)>\alpha_{j^{*}} \Delta_{i\left(j^{*}\right)}(p+1,1)+f_{i\left(j^{*}\right)}\left(x_{1}^{i\left(j^{*}\right)}\right) .
$$

Поэтому в силу неравенств (7) получаем

$$
\alpha_{j} \leqslant \frac{1}{r} \quad \forall j \in N_{k}, \quad \alpha_{j^{*}}<\frac{1}{r} .
$$

Тогда

$$
\sum_{j=1}^{k} \alpha_{j}<\frac{k}{r} \leqslant 1,
$$

но $\left(\alpha_{1}, \alpha_{2}, \ldots, \alpha_{k}\right) \in \Lambda_{k}$. Полученное противоречие доказьвает теорему 1.

Из теоремы 1 вытекают два следствия. 
СлЕДСтвиЕ 1. г-критериальная задача $(X, F)$ с конечным множеством векторных оченок $Y$ разрешима с помощью алгоритма ЛСК, если выполняются равенства $m_{i}=2 \quad \forall i \in N_{r}$.

СлЕДСТВИЕ 2. Для всякой $r$-критериальной задачи $(X, F)$ с конечным множеством векторных оченок $Y \subset \mathbb{R}_{+}^{r}$ и любого вектора $\varkappa_{1}\left(\varkappa_{1}, \varkappa_{2}, \ldots, \varkappa_{r}\right)$ с компонентами

$$
\varkappa_{i} \geqslant \frac{\log _{a} r}{\log _{a} \min \left\{f_{i}\left(x_{j+2}^{i}\right) / f_{i}\left(x_{j+1}^{i}\right): j \in N_{m_{i}-2}\right\}} \quad \forall i \in N_{r}
$$

при а> 1 выполняется равенство

$$
\widetilde{X}=\left\{X(\lambda, \varkappa): \lambda \in \Lambda_{r}\right\}
$$

$2 \partial e$

$$
X(\lambda, \varkappa)=\arg \min \left\{\sum_{i=1}^{r} \lambda_{i}\left(f_{i}(x)\right)^{\varkappa_{i}}: x \in X\right\} .
$$

Отметим, что в [6] был получен аналогичный результат для векторных задач булевого программирования. Однако, предложенная там процедура вычисления нижних оценок для компонент вектора $\varkappa$ является рекуррентной и более трудоемкой.

ЗАмЕчАниЕ 1 . Следующий пример показьвает, что утверждение теоремы 1 перестает быть справедливым, если в ее формулировке неравенства (7) хотя бы для одной пары индексов $i \in N_{r}, p \in N_{m_{i}-2}$ заменить на более слабые неравенства

$$
r \Delta_{i}(p+1,1)-\varepsilon \leqslant \Delta_{i}(p+2,1)
$$

где $\varepsilon>0$.

ПРИМЕР. Пусть $X=\left\{x_{1}, x_{2}, x_{3}\right\}, r=2$,

$$
\begin{gathered}
f_{1}\left(x_{1}\right)=4, \quad f_{1}\left(x_{2}\right)=2, \quad f_{1}\left(x_{3}\right)=0, \\
f_{2}\left(x_{1}\right)=0, \quad f_{2}\left(x_{2}\right)=2, \quad f_{2}\left(x_{3}\right)=4-\varepsilon, \quad 0<\varepsilon<2 .
\end{gathered}
$$

Легко видеть, что для второго критерия $f_{2}(x)$ неравенства $(7)$ не вьполняются, но выполняются неравенства (11). Однако, эта задача неразрешима с помощью алгоритма ЛСК.

2. Разрешимость траекторных задач. Пусть $E=\{e\}-$ конечное множество элементов мощности $n$, а $T=\{t\}$ - некоторая совокупность непустых подмножеств множества $E$, называемьх траекториями. На множестве $E$ зададим векторную весовую функцию

$$
W=\left(w_{1}, w_{2}, \ldots, w_{r}\right): E \rightarrow \mathbb{R}^{r}, \quad r \geqslant 2,
$$

а на множестве траекторий $T$ - векторную целевую функцию

$$
F=\left(f_{1}, f_{2}, \ldots, f_{r}\right): T \rightarrow \mathbb{R}^{r}
$$

частными критериями которой могут быть критерии двух видов:

$(\operatorname{MINMAX}) f_{i}(t)=M\left(w_{i}, t\right)=\max \left\{w_{i}(e): e \in t\right\} \rightarrow \min _{T}$,

$\left(\mathrm{MIN}\right.$ MIN) $f_{i}(t)=N\left(w_{i}, t\right)=\min \left\{w_{i}(e): e \in t\right\} \rightarrow \min _{T}$ 
в любой комбинации.

В дальнейшем, в качестве множества допустимых решений $X=\{x\}$ будем рассматривать множество траекторий $T=\{t\}$, а задачу нахождения паретовского множества $\widetilde{T}$ будем назьвать векторной траекторной задачей порядка $r \times n$ и обозначать через $Z_{n}^{r}(W)=(E, T, W, F)$.

В схему траекторных задач вкладываются многие широко известные задачи оптимизации на графрах, а также задачи булевого программирования (см., например, [8], [9], $[12]-[14])$.

Упорядочим значения весовой функции $w_{i}(e), i \in N_{r}$, на множестве $E$ :

$$
w_{i}\left(e_{1}^{i}\right) \leqslant w_{i}\left(e_{2}^{i}\right) \leqslant \cdots \leqslant w_{i}\left(e_{n}^{i}\right)
$$

Введем следующие обозначения:

$$
\begin{gathered}
q=\min \{|t|: t \in T\} \\
w_{i j}=w_{i}\left(e_{j}^{i}\right) \quad \forall i \in N_{r}, \quad \forall j \in N_{n}, \\
J(i, j)=\left\{k \in N_{n}: w_{i j}<w_{i k}\right\} \quad \forall i \in N_{r}, \quad \forall j \in N_{n} \\
s(i)=\max \left\{j \in N_{n}: J(i, j) \neq \varnothing\right\} \quad \forall i \in N_{r} \\
l(i, j)=\min \{k \in J(i, j)\} \quad \forall i \in N_{r}, \quad \forall j \in N_{s(i)} .
\end{gathered}
$$

Заметим, что в силу условий (6) для любого $i \in N_{r}$ существует индекс $j \in N_{n}$ такой, что $J(i, j) \neq \varnothing$.

Пусть $I_{\mathrm{MAX}}$ и $I_{\mathrm{MIN}}$ - множества тех чисел из $N_{r}$, которыми занумерованы соответственно критерии MIN MAX и MIN MIN.

В силу очевидных включений

$$
\left\{M\left(w_{i}, t\right): t \in T\right\} \subseteq\left\{w_{i j}: q \leqslant j \leqslant n\right\}, \quad\left\{N\left(w_{i}, t\right): t \in T\right\} \subseteq\left\{w_{i j}: j \in N_{n-q+1}\right\}
$$

справедливых для любого индекса $i \in N_{r}$, и теоремы 1 имеет место

ТЕорема 2. Для того чтобы векторная траекторная задача $Z_{n}^{r}(W)$ была разрешима с помощью алгоритма ЛСК достаточно, чтобы выполнялись неравенствa

$$
\begin{array}{ll}
w_{i, l(i, j)} \geqslant r w_{i j}-(r-1) w_{i q} & \forall i \in I_{\mathrm{MAX}}, \quad \forall j \in N_{s(i)} \backslash N_{l(i, q)-1} \\
w_{i, l(i, j)} \geqslant r w_{i j}-(r-1) w_{i 1} & \forall i \in I_{\mathrm{MIN}}, \quad j=2,3, \ldots, \min \{n-q, s(i)\} .
\end{array}
$$

3. Алгоритм сведения к разрешимой задаче. В [15] был анонсирован алгоритм сведения любой векторной траекторной задачи с одним критерием произвольной природы и несколькими критериями вида MINMAX к эквивалентной задаче (с тем же паретовским множеством), разрешимой с помощью алгоритма ЛСК. Ниже приведем алгоритм $\varphi$, сводящий любую векторную траекторную задачу $Z_{n}^{r}(W)=(E, T, W, F)$ к эквивалентной векторной задаче $Z_{n}^{r}\left(W^{\prime}\right)=\left(E, T, W^{\prime}, F\right)$, разрешимой с помощью алгоритма ЛСК. 
Алгоритм $\varphi$.

Этап 1. Для любого $i \in N_{r}$ упорядочим весовые функции $w_{i}(e)$ на множестве $E$ (cм. (12)).

Этап 2 состоит из $r$ шагов, причем на $i$-м шаге, $i \in N_{r}$, строится новая весовая функция $w_{i}^{\prime}(e), e \in E$. Каждьй $i$-й шаг содержит $n$ подшагов.

$k$-й подшаг $i$-го шага, $k \in N_{n}, i \in N_{r}$.

Если $k \leqslant l(i, \pi)$, то полагаем $w_{i}^{\prime}\left(e_{k}^{i}\right)=w_{i}\left(e_{k}^{i}\right)$, где

$$
\pi= \begin{cases}q, & i \in I_{\mathrm{MAX}} \\ 1, & i \in I_{\mathrm{MIN}}\end{cases}
$$

Если $l(i, \pi)<k \leqslant \rho$, то

$$
w_{i}^{\prime}\left(e_{k}^{i}\right)= \begin{cases}w_{i}^{\prime}\left(e_{k-1}^{i}\right) & \text { при } \delta_{i}(k, k-1)=0 \\ w_{i}^{\prime}\left(e_{\pi}^{i}\right)+r \delta_{i}^{\prime}(k-1, \pi) & \text { при } \delta_{i}(k, k-1)>0\end{cases}
$$

где

$$
\rho=\left\{\begin{array}{ll}
n, & i \in I_{\mathrm{MAX}}, \\
n-q+1, & i \in I_{\mathrm{MIN}},
\end{array} \quad \delta_{i}(j, l)=w_{i}\left(e_{j}^{i}\right)-w_{i}\left(e_{l}^{i}\right), \quad \delta_{i}^{\prime}(j, l)=w_{i}^{\prime}\left(e_{j}^{i}\right)-w_{i}^{\prime}\left(e_{l}^{i}\right)\right.
$$

Если $\rho<k \leqslant n$, то $w_{i}^{\prime}\left(e_{k}^{i}\right)=w_{i}^{\prime}\left(e_{k-1}^{i}\right)$.

Нетрудно убедиться, что для задачи $Z_{n}^{r}\left(W^{\prime}\right)=\left(E, T, W^{\prime}, F\right), W^{\prime}(e)=\left(w_{1}^{\prime}(e), w_{2}^{\prime}(e)\right.$, $\left.\ldots, w_{r}^{\prime}(e)\right)$, выполняются условия теоремы 2 , а паретовские множества задач $Z_{n}^{r}(W)$ и $Z_{n}^{r}\left(W^{\prime}\right)$ совпадают.

Очевидно, что трудоемкость алгоритма $\varphi$ оценивается величиной $O\left(r n \log _{2} n\right)$.

Следовательно, справедлива

ТЕорема 3. Алгоритм ч трудоемкости $O\left(r n \log _{2} n\right)$ сводит любую векторную траекторную задачу $Z_{n}^{r}(W) \kappa$ әквивалентной векторной траекторной задаче того же порядка, разрешимой с помощью алгоритма ЛСК.

ЗАмЕчаниЕ 2. Легко видеть, что $r$-критериальная задача $(X, F)$ с конечным множеством допустимых решений $X$ и критериями произвольной природы эквивалентна векторной траекторной задаче $Z_{n}^{r}(W)=(E, T, W, F)$ с критериями MIN MIN $\left(N_{r}=I_{\mathrm{MIN}}\right)$, где $n=|X|$,

$$
\begin{gathered}
E=T=N_{n}, \quad X=\left\{x_{1}, x_{2}, \ldots, x_{n}\right\}, \\
W(e)=\left(w_{1}(e), w_{2}(e), \ldots, w_{r}(e)\right), \quad w_{i}(e)=f_{i}\left(x_{e}\right) \quad \forall i \in N_{r}, \quad \forall e \in E, \\
F=\left(f_{1}, f_{2}, \ldots, f_{r}\right): T \rightarrow \mathbb{R}^{r}, \quad f_{i}(t)=\min \left\{w_{i}(e): e \in t\right\} \rightarrow \min _{T} .
\end{gathered}
$$

Поэтому, если для $r$-критериальной задачи $(X, F)$ с конечньм множеством допустимых решений $X$ неравенства (7) не вьполняются, то ее можно свести к эквивалентной траекторной задаче $Z_{n}^{r}\left(W^{\prime}\right)$ с критериями MIN MIN, разрешимой с помощью алгоритма ЛСК. 


\section{СПИСОК ЦИТИРОВАННОЙ ЛИТЕРАТУРЫ}

[1] Емеличев В.А., Кравцов М.К., Янушкевич О.А. Лексикографические оптимумы многокритериальной задачи дискретной оптимизации // Матем. заметки. 1995. Т. 58. № 3 . C. $365-371$.

[2] Geoffrion A. M. Proper efficiency and the theory of vector maximization // J. Math. Anal. Appl. 1968. V. 22. P. 618-630.

[3] Михалевич В. С., Волкович В. Л. Вычислительные методы исследования и проектирования сложных систем. М.: Наука, 1982.

[4] Подиновский В. В., Ногин В. Д. Парето-оптимальные решения многокритериальных задач. М.: Наука, 1982.

[5] Дубов Ю.А., Травкин С. И., Якимец В. Н. Многокритериальные модели формирования и выбора вариантов систем. М.: Наука, 1986.

[6] Burkard R.E., Keiding H., Krarup J., Pruzan P. M. A relationship between optimality and efficiency in multicriteria $0-1$ programming problems // Comput. Oper. Res. 1981. V. 8. № 4. P. 241-247.

[7] Емеличев В. А., Перепелица В.А.Сложность дискретных многокритериальных задач // Дискретная матем. 1994. Т. 6. №1. С. 3-33.

[8] Емеличев В. А., Кравцов М.К. О неразрешимости векторных задач дискретной оптимизации на системах подмножеств в классе алгоритмов линейной свертки критериев // Докл. PAH. 1994. T. 334. № 1. C. 9-11.

[9] Емеличев В.А., Кравцов М.К. О задачах векторной дискретной оптимизации на системах подмножеств, неразрешимых с помощью алгоритмов линейной свертки // ЖВМиМФ. 1994. T. 34. № 7. C. 1082-1094.

[10] Емеличев В. А., Ковалёв М. М., Кравцов М. К. Многогранники, графы, оптимизация. М.: Наука, 1981.

[11] Гладкий А.А., Янушкевич О.А. О линейной свертке частных критериев векторной задачи минимизации // IX Всероссийская конференция "Математическое программирование и приложения". Тезисы докладов. Екатеринбург, 1995. С. 67.

[12] Пападимитриу X., Стайглиц К. Комбинаторная оптимизация, алгоритмы и сложность. М.: Мир, 1985.

[13] Емеличев В.А., Кравцов М. К. О комбинаторных задачах векторной оптимизации // Дискретная матем. 1995. Т. 7. №1. С. 3-18.

[14] Леонтьев В.К., Гордеев Э.Н. Качественное исследование траекторных задач // Кибернетика. 1986. № 5. С. 82-89.

[15] Yemelichev V.A., Kravtsov M. K., Yanushkevich O. A. On solvability of vector problems on systems of subsets using a linear criteria convolution algorithm // 2nd International Conference "Mathematical Algorithms". Abstracts. Nizhny Novgorod, 1995. P. 21.

БелНИИ экономики и информатики ААН РБ, г. Минск

Поступило

Институт технической кибернетики АН РБ, г. Минск

14.08.95 